\title{
A methodological approach for a home occupants centred web tool to support buildings energy retrofitting process
}

\author{
Stefano Pili ${ }^{1}$, Francesca Poggi ${ }^{1}$ and Caterina Frau $^{1}$ \\ ${ }^{1}$ Sotacarbo SPA, Grande Miniera di Serbariu, 09013 Carbonia (SU), Italy
}

\begin{abstract}
This paper presents a methodological approach to develop a user-oriented web tool to support the early stage of the decision making process for energy retrofitting of residential real estates. The tool mixes a simple analytical Urban Building Energy Model (UBEM) to user-oriented feedback interfaces to simulate the retrofitting potential of the residential units. The AUREE tool will be based on a Web - GIS GeoBlog portal with customized interfaces aimed to share the knowledge on urban building heritage and promote participation of stakeholders of the urban community. There are many literature exempla of guidelines or quality protocols on the topic of energy efficiency specified to particular types of buildings or focused on a territorial area, but these are generally not integrated into a geographically based tool. Moreover, the AUREE methodology aims to set interfaces that could use the portal users' feedback to update the content of the portal itself. After touch on the theoretical context, a summary of the AUREE project is presented, and then the paper focuses on the structures of algorithms and tools adopted on the residential users' interfaces. The interface mixes the results of an engineering Urban Building Energy Model based on local archetypal approach and standard energy calculation (series UNI 11300 - TS), with participative data provided via user-interactive interfaces to obtain customized retrofitting scenarios and suggestions. At the end of the paper, the main critical aspects and the further step of the research project are pointed
\end{abstract}

\section{Introduction}

While Europe's buildings are unique and diverse and reflect the culture of our continent, many of them are also old and inefficient(about $200 \mathrm{kWh} / \mathrm{m} 2$ per year) [1]. More than 220 million buildings, representing approximately $85 \%$ of the building stock, were built before 2001 and will mostly still be standing in 2050 . Buildings are responsible for about $40 \%$ of total energy consumption in the EU and $36 \%$ of greenhouse gas emissions from energy. Building renovation is crucial to tackling this energy use and emissions, to meeting the EU's 2030 emission reduction target and to becoming climate neutral by 2050, as well to increase resilience to climate impacts.

In line with a net 55\% emission reduction target, the EU Commission expects the actions in the Renovation Wave to reduce buildings' greenhouse gas emissions by $60 \%$ (from $456 \mathrm{MtCO} 2 \mathrm{eq}$ to $161 \mathrm{MtCO} 2 \mathrm{eq}$ ), their final energy consumption by $14 \%$ (from 374 $\mathrm{MtCO} 2 \mathrm{eq}$ to $321 \mathrm{MtCO} 2 \mathrm{eq}$ ) and energy consumption for heating and cooling by $18 \%$ (from $318 \mathrm{MtCO} 2$ eq to $259 \mathrm{MtCO}$ eq) as compared to 2015.

\footnotetext{
${ }^{1}$ Corresponding author: stefano.pili@sotacarbo.it
} 
Building renovation is one of the sectors facing the largest investment gap in the EU. To achieve the proposed $55 \%$ climate target by 2030 , around $€ 275$ billion of additional investment in building renovation is needed every year.

Today, renovation reduces energy consumption of the buildings by only $1 \%$ per year. Deep renovations, that improve the energy performance of a building by at least $60 \%$, are annually carried out only in $0.2 \%$ of the building stock and, in only a fifth of the cases, energy efficiency is significantly improved.

The Renovation Wave aims for more and deeper renovation. The Commissions' target is to at least double the average of energy renovation rate by 2030 , while increase the average gains in term of energy efficiency. This could lead to renovate 35 million buildings in the coming decade. This can trigger a virtuous cycle between higher demand for deeper renovation and falling costs for smarted and more sustainable products and simpler and faster renovation processes. Renovated homes that are energy and resource efficient will reduce energy bills while improving health, comfort and wellbeing.In addition to its ecological benefits, building renovation creates local jobs and stimulates much-needed investments in economy.

Renovation can be held back by barriers at different points in the renovation value chain from the initial decision to engage in renovation, to financing and completion of the project. For instance, when considering a renovation, benefits from energy savings might be uncertain or poorly explained and understood, especially by the end-users. They can be difficult to measure and monetise. Renovating can also be costly, difficult to organise and lengthy to carry out. Mobilising financing can be difficult, in particular at local and regional level. Public funds are frequently scarce and difficult to blend due to regulatory obstacles and lacking capacity in public administrations.

\section{Theoretical context}

There are many literature exempla of guidelines or quality protocols about energy efficiency enhancement of building heritage, some of them are also addressed to specific kind of buildings (historical, school, offices ...) [2] [3].These are generally documents developed for a technical audience thatsometimes have difficulty in spreading even among operators in the construction industry and are almost unknown to non-technical citizens. The lack of shared knowledge between construction industry operators and non-technical actors about the building heritage is considered one of the main barriers in the recovery process [4].In particular, the main decision-makers in a building renovation are often nontechnical actors (owners of the houses, politicians for PA buildings ...) who, in most cases do not have the expertise even to make the right questions in the first step of the design process.

The development and experimentation of tools to support the creation of shared knowledge among the actors involved in a process of heritage renewal is becoming a central theme to favour the process. This type of tools, generally based on more or less freely accessible web interfaces, support the first design-planning phases and can be addressed both to technicians and to non-technical users. These tools ask the user to input data or, more or less structured information and in exchange provide knowledge about the real estate unit retrofit potential or about the use of renewable sources. With the purpose to create a shared framework at European scale, the ENERFUND [5] uses regional data from Energy Performance Certificates to set up a comparison tool between buildings, based on a geographic interface, aimed at explaining their retrofitting potential. With a similar purpose, another European project [6] developed for some Finnish cities presents a geographical interface to select a building that shows energy performance values based on local average values. Through simple interfaces, a user can correct information on the 
building systems and envelope, obtaining the potential retrofit of common technologies. Finally, if interested, he can contact local companies registered on the portal. Compared to the previous one, it requires more user effort to get to result, but offers more personalized suggestions. Some web tools aim mainly to communicate and share best practices. These type of portals collect and represent information about energy performance and/or energy consumption at single building scale and make comparison to building stock stats of some Italian and European cities [7] [8] [9]. Other tools [10], often aimed at commercial purposes [11], focus instead on the evaluation of the single real estate unit and not at the comparison between buildings. Therefore, they generally lack a geographical interface, but require a more complex set of information to describe the building systems. The Bologna Solar City portal [12] on the other hand, is dedicated to the study of solar technologies potential (thermal and photovoltaic). The portal user can select the roof of a building from a cartographic base and, through simple questions, could be able to evaluate its theoretical, technical and economic potential. Other tools are more addressed at technical audience to support project's preliminary phases at the building scale or for energies communities. ENEA has developed apps to support the survey and the feasibility assessment of schools and condominiums [13]. These tools provide brief suggestions and an assessment of the energy retrofitting potential, adopting simplified algorithm based on data imputed by the user about building characteristics and the energy bills that are similar to a first level Energy Audit. ENEA has been developing and testing some web based tools to support in feasibility assessment and in preliminary project of a energy communities grid configuration [14] [15].This tools adopt highly simplified data about building structure and systems, but require more detailed inputs on energy consumption profiles based on typology of real estate occupants that could require a quite specialised technical users to be used.

One of the most critical aspects of these web based tools is the need to find a balance between the simplification of concepts aimed at share knowledge with the targeted stakeholders without losing significance and richness of contents. Many of these tools addressed to not technical stakeholders perform simplified calculations based on average parameters for climatic zone or for smaller territorial contexts (region, province, more rarely city) which generally do not consider geometric factors or context that can strongly influence energy needs. This is even more evident in the evaluation of the economic feasibility of interventions that is often based on highly arbitrary cost factors that not considers building characteristics (shape, context...) and are not linked to the peculiarities of local building stock. When the tool is able to perform more detailed calculation, a not negligible amount of input data is often required, limiting the audience of the tool to technical users. The AUREE methodology try to set up a tool targeted to homeowners (not technical audience) that, asking some basic data about real estate characteristics, can give specific retrofit suggestion based on a specifically developed study about local typologies and building geometries provided from topographical data.

Thanks to the diffusion of new technologies and the greater availability of geographic data, the development of Urban Building Energy Modelling (UBEM) tools [16] is becoming an interesting field of research. UBEM are tools mainly oriented to urban or suburban scale that incorporate the geographical dimension of information useful for relating to the other traditional urban planning typical themes and to spatial represent the energy demand. These tools are able to link the project of single building with the urban and neighbourhood scale, oriented to the development of energy communities and to support the definition of sustainable development strategies in a broader sense.

In general, these tools are able to calculate the buildings energy needs and provide procedures to estimate the retrofit potential of technologies improvements and/or design scenarios [17]. They differ significantly from each other in terms of availability of the basic 
data, reliability and detail (temporal, spatial, etc.) of the results, complexity of the algorithms and type of approaches to modelling (top down, bottom up, etc.). One of the main differences is the type of approach for the energy calculation [18] [19]: statistical, engineering-analytical or mixed. Statistical models use different predictive inferential techniques to derive energy demand from a group of known variables (type of buildings, user profile, time series of consumption of a sample of units ...). These models can represent with good reliability the trend of energy demand as they implicitly incorporate the "human" variable, but show limits in the evaluation of technological innovations effects as they are not representative of physical phenomena. The main lack point of these models is that the energy consumption data required for the statistical modelling are not always available. On the contrary, analytical models can simulate theoretical energy needs starting from the physical characteristics of the heritage (geometries, materials, plants, etc.), using simulative models based on climatic models. Many literature examples in order to exploit the strengths of both calculation methods, adopt mixed approaches that involve the use of simulation for a real buildings sample or for building types at the beginning and then the use of statistics techniques to up-scale the results to all the building stock [20].

In general, complex models, according to a better reliability of the results, require more data and onerous activities of results analysis. Less complex models adopt several simplifying hypotheses, therefore they require fewer resources, and have usually more repeatable procedures. However, the uncertainty of initial assumptions in this case burdens the results [21]. One of the most used simplifying approaches adopted by these tools is the"building archetype" that represent the most common characteristic of the local heritage related to the energy need and retrofit potential. The archetype characterisation is generally based on a significant sample of buildings and then applied to the whole city via appropriate methods (analytical, statistical ...) to extending the results of the sample to all similar buildings in the city. The Tabula EU Project [22] adopts an archetypal approach based on energy standard calculation in order to estimate the energy retrofit potential of the European building heritage. It defines typologies for each national climate zones based on year of built and building typology. The retrofit potential has been studied for each typology and then scaled to national level by census stats on building heritage.

Others approaches develop a mixed calculation approach with simulation and statistical techniques to calibrate the model on energy consumption data [23] [24]. These models generally adopt a few basic data for the building stock physical characterisation, but thanks to appropriate calibration, methodologies could ensure a good estimation of the energy demand [24]. This approach could limits the effort for preliminary data treatment and for survey activities, but physical characteristics of the heritage that could direct affect the renovation and energy retrofit potential could be missed. Moreover, in the majority of the Italian urban contexts, mostly in the average - small sized cities, energy consumption data are not available and unlikely could have enough resources to involve highly specialized expertise in managing complex software. Common critical aspects of the UBEM approach are the availability of trustable base data with proper spatial and time details, the complexity of procedures and tools that could limit the replication and readability of the methodologies, and the results reliability and meaningfulness for decision makers. Therefore, the definition of a widespread methodology that could be integrated on the more common urban planning practice is still an open field of research.

The concept of the AUREE tool is trying to fill the knowledge gap between the stakeholders involved on the heritage renovation process by integrating, in a Geographical Information System (GIS) environment, a building archetypes study of local city heritage with a participative web based interfaces that aim to improve the archetype characterisation itself. Moreover, the methodology proposes also an easy UBEM approach aimed to improve the reliability of the web tool results and suggestions. 


\section{Methodology: general framework}

The AUREE Project (Urban Energy Abacus of Buildings) aims to develop and experiment a support tool for energy efficiencyimprovement and renovationof the urban building heritage based on a portal (Web - GIS, GeoBlog). The web tool contains specific interfaces aimed at sharing the knowledge of building stock and promoting participation of local stakeholders: occupants of the real estate units, decision makers of the Public Administration, construction industry operators or other actors with a more generic interest. The methodologyis designed for small and medium-sized Italian cities and is based on information sources commonly available in the national territory such as geographical OPEN data, knowledge of the building heritage linked to local urban planning and thematic disciplinary references (technical regulations, scientific studies and gray literature). These data may be integrated with expeditious urban survey activities that are commonly implemented in the modern urban planning practices.

The methodology therefore consists of an analysis and representation protocol of the building heritage on a geographical basis, combined with a tool for communication and involvement of local actors developed on the WEB GIS portal organized in three main sections containing the geographical information and specific interfaces for interaction with local actors (fig. 1):

- Public buildings: it constitutes a support tool for energy management of the public buildings but also a tool for sharing the values (public services, historic, ...) of the public buildings heritage and to promote the transparency of energy uses;

- Residential heritage: contains the spatial representation of the energy performance of the building heritage and aims to directly involve the occupants in providing information on their home in order to have suggestions for specific retrofits in exchange;

- Geographical Abacus: collects and reorders knowledge on recurring elements of the building heritage on a GIS framework, favouring its dissemination among construction industry operators but also to a non-technical audience.

In addition to these sections connected to specific interfaces, it is then possible to consider all the context layers, even if not directly linked to the aspects of energy efficiency, which can however influence the renovation potential (regulations, demography data).This open set of themescreates a shared background knowledge among decision makers and other stakeholders to direct towards the development of holistic approaches to urban planning.

In the case of public buildings, a protocol based on energy audit procedures and sensors monitoring is proposed. This approach aims to represent and share the energy related data of Public building (transparency of public energy uses). In addition,an energy management interfaces based on BIM and sensors integration will be developed and tested. The representation of the energy characteristics of the residential heritage is based on the study of building typologies and its recurring elements (Abacus) coupled with the development of an energy model at the urban scale (Urban Building Energy Modelling UBEM), useful for preliminary evaluation of the retrofit potential of the building stock.

The Abacus and the Residential Heritage sections are strongly linked, because these are different representations of the same set of information based on: typological studies, UBEM results and data from participatory interfaces. The abacus is a spatial database that contains the typological information of the building heritage linked to the geographical reference element (the building footprint). The concept is trying to fill the knowledge gap between the stakeholders involved on the heritage renovation process by integrating a 
building archetypes study of the city heritage with participative web based interfaces that are aimed to improve the archetype characterisation itself.

One of the AUREE project purposes is to develop and test an archetypal approach at local scale based on commonly available geographical data and easy urban surveys. The methodology has been tested on the Carbonia's municipality, but most of the typologies that has been defined could be used also in describing the whole Sardinian building stock.

The research purpose is therefore to define in detail the procedures and tools that connect the different thematic contents (public buildings layer, building heritage layer, geographical abacus, context layer, data collected by the sensors) to the representation and interaction with local actors through the portal (see fig.1.).

This article focuses to the methodological approach for the definition of the home occupant interface coupled with the UBEM approach, leaving the detailed description of the UBEM algorithms and of the other elements of the AUREE project to further publications.

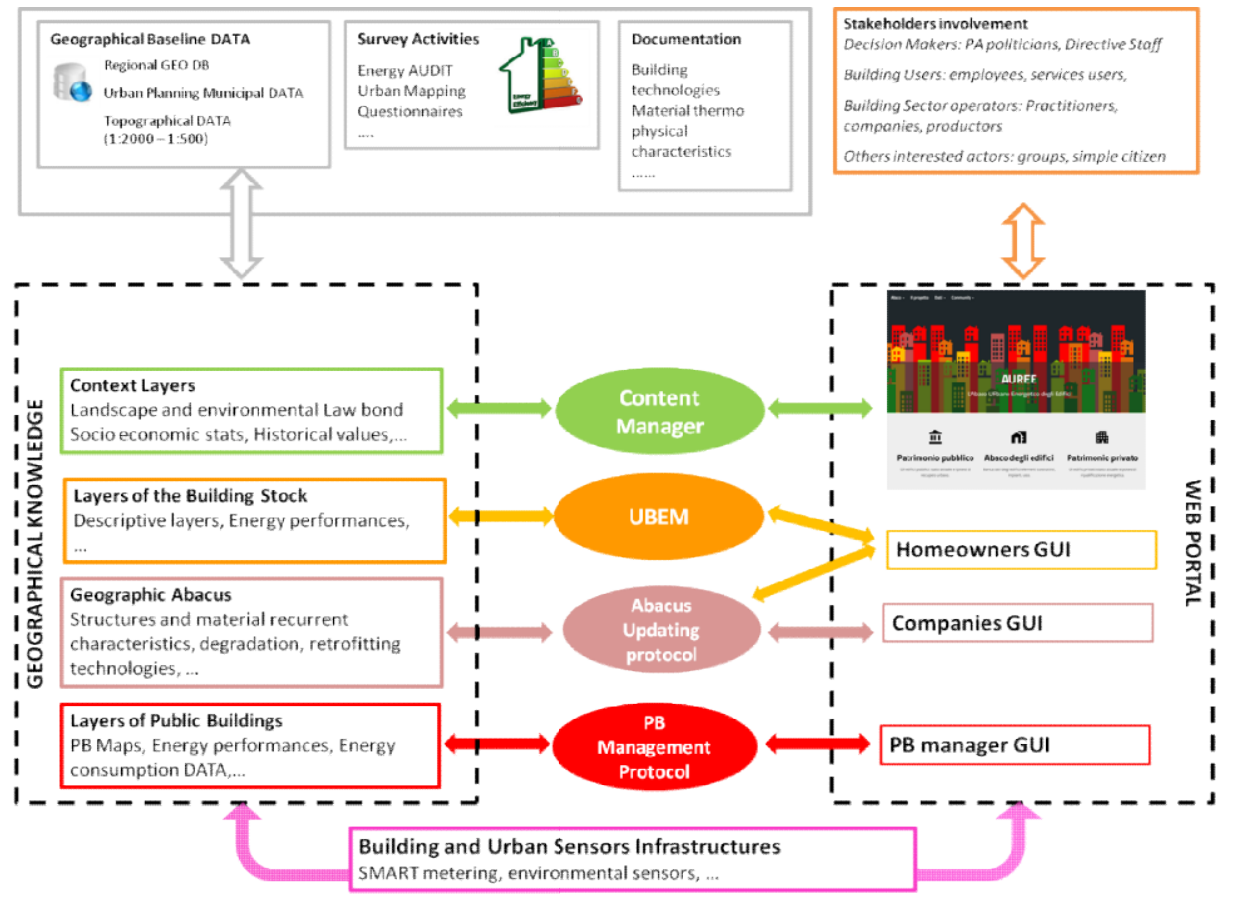

Fig.1. AUREE framework

\section{The UBEM methodological approach}

The methodology is based on the evidence that in medium-small urbancontexts with a Mediterranean climate there is a strong lack of spatially detailed data on the energy consumption of the building stock. Urban energy services networks do not serve these kind of cities often and the autonomous heating systems, which are characterized by a great variety of technologies and energy carriers, is far more widespread than the centralized one. Furthermore, the incidence of real estate units without a real heating system continues to be not negligible. Mostly these use traditional systems such could be fireplaces, electric and gas stoves. 
The proposed methodology has been designed considering the lack of energy consumption data, and also has been adapted to the covid-related restrictions regarding onsite surveys in residential units and offices. The model developed for the AUREE project has a dual role. In addition to the typical purpose of these tools, namely the estimation of the energy needs of the building stock and the definition of possible retrofit scenarios, the model constitutes the base information to allow interaction with the owner of the residential real estates. Taking into account the lack of energy consumption data necessary to optimize the reliability of the model through calibration, the model makes preliminary a performance calculation consistent to standardsbased on typological hypotheses. Use this calculation give a common reference for the involved stakeholders and cloudfor the quantitativequalitative evaluation of the potential of the energy retrofit.Moreover, the standard calculation is commonly adopted to assess the energy performance law requirement of a renovation project ad to verify the possibility to use incentives. The typological standard calculation will be then refined by data provided by the interaction with the home occupant, who can provide information about building structures and systems and data about energy consumption useful to calibrate the estimation.Using the GIS data processing capabilities, a simple archetype based engineering UBEM has been set up. It is able to calculate the energy performance of each building on study area adopting:

- the geometries obtainable for each building from the topographic basis (dispersing surfaces, volumes ...) via GIS procedures;

- the typological characteristics of the building envelope and of the more common plant systems on the local heritage;

- a simplification of the calculation algorithms proposed by current technical standards (UNI TS 11300 series) [26]

In this way, thematic maps relating to the energy performance of the heritage are obtained, the occupants of the homes, after a profile registrations, could use it to access a specific interface. The interface shows the information of the typological study and the preliminary results of UBEM, but asks the user to answer a questionnaire concerning characteristics of his real estate unit and his profile of use. These answers will be combined to the UBEM results both to generate advice for specific energy retrofit actions and to modify the statistics that are the basis of the UBEM itself and the contents of the Abacus (for more detail on questionnaire see https://www.auree.it/).

The final version of the portal will allow users to:

- make a comparison between your own energy consumption data and those of similar buildings;

- make a comparison between your own energy consumption data and the results of simulative model;

- be aware of the opportunities and limitations of your building;

- obtain advice for your home on possible retrofit actions aimed at saving energy, reducing costs and improving comfort;

- obtain addresses and contacts of local companies.

The result of the calculation of the envelope energy need of each building will then be aggregated by spatial ISTAT census section. Together with others ISTAT data and the statistics deriving from the interactions of the building occupants with the portal, the energy needs and the theoretical demand of the sector will be calculated.

The evaluation of the reliability of the models and procedures will be assessed on the results of the owners interface and on the urban scale calculation. In the first case, a comparison will be made between the results of the model, the participatory data referring to energy consumption and those obtainable through control software set up on typical 
cases. The calculation at the neighbourhood scale, characterized by a greater degree of indeterminacy, will be evaluated by comparing the data obtained through the portal and the average consumption profiles defined by the literature and by known parameters on a regional basis.

In order to better support the definition of the energy modelling, an energy and comfort indoor motoring campaign in some Carbonia's buildings where planned. Unfortunately, due to the pandemic restrictions this activity has been delayed for further research, when the participative data from the portal could be compared to the measured ones. Moreover,the development of AUREE project for the Public Building is testing SMART sensors for indoor environment and energy consumption monitoring that are integrated in to the portal for data visualization and sharing. In the further research, a simplified version of this technology could be tested for use on residential real estates and compared to some available commercial solutions and, of course, to the questionnaire based data. 


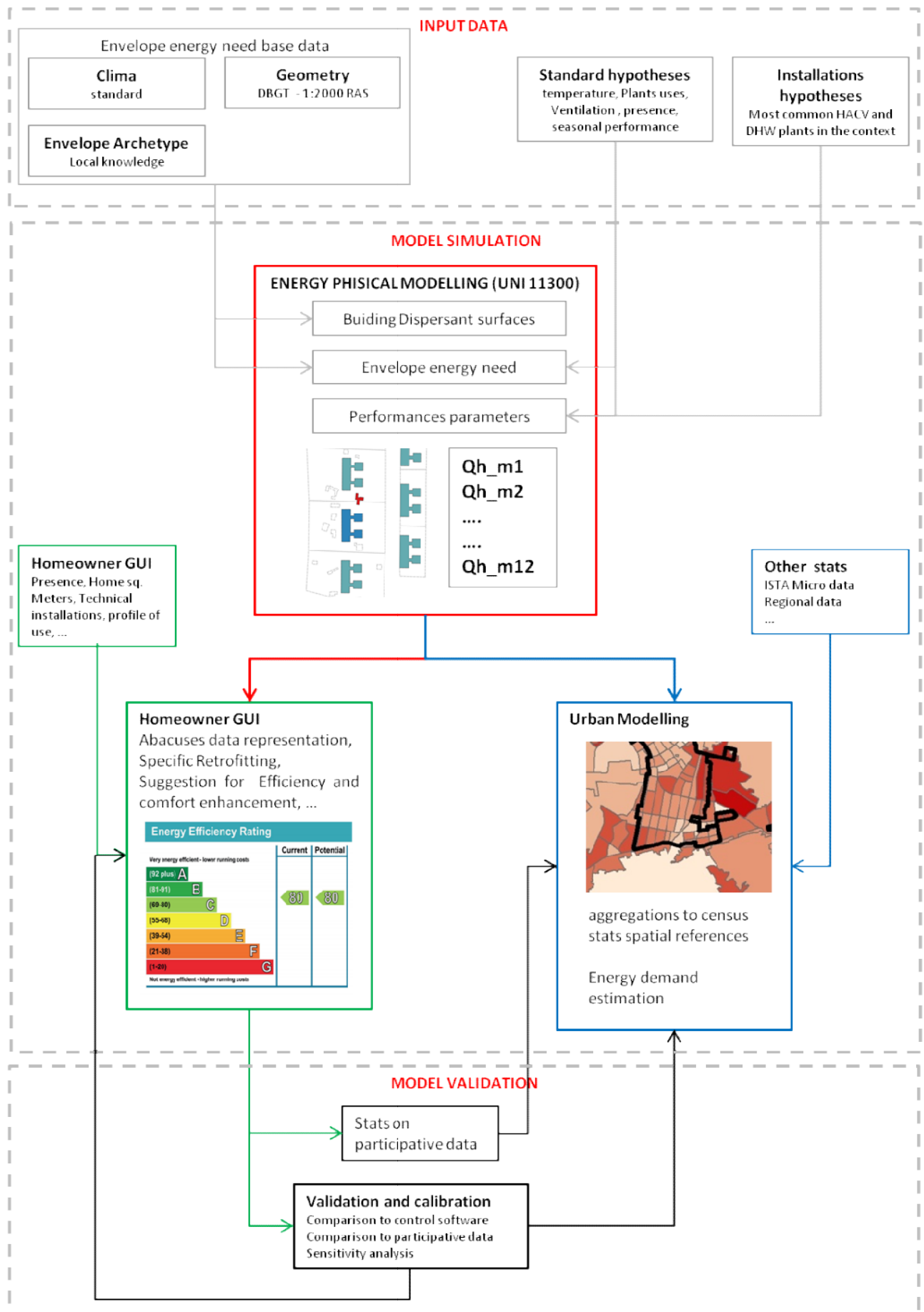

Fig. 2. The framework of the Urban Building Energy Model 


\section{The methodological approach for the Home occupants interface}

The questionnaire is mainly addressed at the citizens of Carbonia wherethe results of UBEM developed in the project are available for each building. This information are the baseline data that users could see in the Carbonia's buildings, but the questionnaire isalso accessible to all users who register on the AUREE platform and in this casethey will find the average information related to the building type in place of those calculated by the model. In this contest, the citizen plays a key role and the data obtained through the questionnaire will make it possible to better understand characteristics of the building heritage, such could be most common technological systems for air conditioning and/ or for DHW the production, energy povertyrelated aspects, use profile, renovations expectations and more. The questionnaire is divided into some main sections:

- General information on the building where the property is located (location, building typology, period of construction, indoor area, ...);

- Building envelope data (types of dispersing surfaces, previous retrofit interventions, windows characteristics ...);

- Home plants technologies (heating, cooling, DHW, RES);

- Plants usage profile (time of use, portion of the house with air conditioning systems, set point temperatures ...);

- Other data (comfort, energy consumption, systems obsolescence, occupant profile, willingness to invest and general satisfaction of the property).

The structure of the questionnaire and the questions themselves has been tested and developed involving a group (about 35 people) of local building owners. The first version of the questionnaire was a form similar to a simplified Energy Audit checklist with prefilled options about structures, plant systems and perceived comfort. The collected answers contribute in the local archetypes definitions and the participant's feedback was used to improve the questionnaire contents and structure. Some people found problems in the choice of the structure type from the form options (about $40 \%$ of the participants) and in the definition of the sub systems of the plants (about $60 \%$ of the participants). Therefore, the definitive questionnaire do not asks about envelope material that are assigned in the archetypes study by year of construction and adopts only a synthetic and simple definition of systems for building energy services: heating, cooling, DHW. As well known in literature for this kind of tools, the most critical questions are those about energy consumption where a few of participants was able to autonomous collect bills data as asked. Considering the main purpose of the tool, the approach for the on line form is to ask a perceived range of expenditure for energy services. It will be used to give appropriate retrofit suggestion by comparing the energy expenditure declared to some reference energy consumption indexes calculated adopting the standard calculation hypotheses and the answers about the profile of use and internal comfort.

The GIS tool performs all the standard calculation steps (UNI 11300 series) and could provide someof the energy performances parameters of the legislation in force. The procedure implemented in the interface starts from the net envelope energy need calculated by the UBEM for the whole building structure. If the building typology implies the presence of apartments at different floor levels, the UBEM calculates a specific envelope energy need for real estate units located on the top floor, on the ground floor or in an intermediate floor.

After having explored the predefined typological data for the selected building, the user can answer general questions regarding the building and his home, allowing identifying the type of apartment (step 1). The answer about the building envelope allow to adapt the needs of the envelope by entering the typical transmittance values of the building 
elements, specified by the user, like the windows and shutters types, any previous retrofit interventions and the occurrence of dispersing surfaces towards air-conditioned or non-airconditioned rooms.

The envelope energy need could be modified adopting the dispersing surfaces, calculated by GIS tools, and the difference in transmittance between the materials hypothesized in the typological study and those identifiable by the users' responses (step 2).

The delivered energy and the consequent parameters linked to the energy vector $(\mathrm{CO} 2$, operating cost, ...) will therefore be calculated (step 3) using the average thermal yields of the selected plant technologies, assuming a standard usage profile (set point temperature for heating / cooling $20^{\circ} / 26$, continuous regime, ..). The same procedure will then be performed, through the usage profile data specified by the user (air-conditioned indoor surface, time of use of the plants, ..) which affect both the envelope energy need and the yields of the plant systems.(step 4)

Then some retrofit scenarios will be proposed, summarized according to a light and a heavy intervention logic, using the energy performance results obtained with the standard profile and by the user customized one. The questionnaire more qualitative answers will help to provide advices to users that could be related to: the project of apartment renovation, improvement of the indoor comfort, investments and access to economic incentives.

The Abacus Geo - DB allowsalso to set up a simple dynamic tool for the preliminary assessment of the economic feasibility of retrofit interventions other than those suggested, which however takes into account the specific condition of the real estate unit that can be summarized by the geometric structural data obtained from UBEM and from the questionnaire answers.

The procedures and algorithms details, especially those of the step 4 and the decision support tool for the identification of the retrofit scenarios are still being defined and will be developed in the next year of research.In order to experiment as soon as possible the interface with users, a simplified calculation procedure (1) (2) (3) will be used for the execution of the first three steps dedicated to adjusting the performance calculation to the users'answers.

All the thermal factors related to the ventilation and use of the rooms are the same for all homes and depend only on the indoor area of the rooms. The solar heat gain is inherently considered on the envelope energy need calculated for the apartments at different floor levels. So for this phase aimed to testing the interface, a simplified procedure is proposed that considers only the unitary envelope energy need variation related to the heat flow through the structures, that could be roughly assessed by the difference of the $U$ values. Since the internal plan of the apartment is not known, the energy need of the envelope of the entire building or of the identified section (ground floor, top floor...) will be calculated with (1). Subsequently, the unit value obtained will be multiplied by the useful area declared in the questionnaire to obtain the values of the apartment (2) (3). 
$Q_{h}{ }^{\prime}=Q_{h}-0,024 * D D\left[\operatorname{tr} * A_{r} *\left(U_{r}-U_{r}{ }^{\prime}\right)+A_{w} *\left(U_{w}-U_{w}{ }^{\prime}\right)+\operatorname{ta} * A_{b} *\right.$

$\left.\left(U_{b}-U_{b}{ }^{\prime}\right)+A_{w i} *\left(U_{w i}-U_{w i}{ }^{\prime}\right)\right](1)$

Where:

$Q_{h}=$ envelope energy need of the apartment (detached, ground floor...) calculated by UBEM procedure

$Q_{h}{ }^{\prime}=$ envelope energy need actual state

$D D=$ local degree days

$A_{r}, A_{w}, A_{b}, A_{w i}=$ structures dispersant surfaces(roof, wall, base, windows) calculated by the UBEM for specific position of the apartment (detached, ground floor...)

$U_{r}, U_{w}, U_{b}, U_{w i}=U$ value of the structure defined by typological study

$U_{r}{ }^{\prime}, U_{w}{ }^{\prime}, U_{b}{ }^{\prime}, U_{w i}{ }^{\prime}=U$ value of the structure defined by questionnaire

tr, ta $=$ heat transmission factor for surfaces (roof, base) to not air conditioned rooms

$Q h_{\text {sta }}=S_{\text {apar }} / S_{\text {build }} * Q h^{\prime}(2)$

$Q d h_{\text {sta }}=Q h_{\text {sta }} / \eta^{\prime}(3)$

Where:

$Q h_{\text {sta }}=$ apartment standard envelope energy need

$Q d h_{\text {sta }}=$ apartment standard delivered energy need

$S_{\text {apar }}=$ apartment indoor surfaces from questionnaire

$S_{\text {build }}=$ indoor surface of the building considered in the UBEM calculation

$\eta^{\prime}=$ thermal yields of the heating system selected by questionnaire 


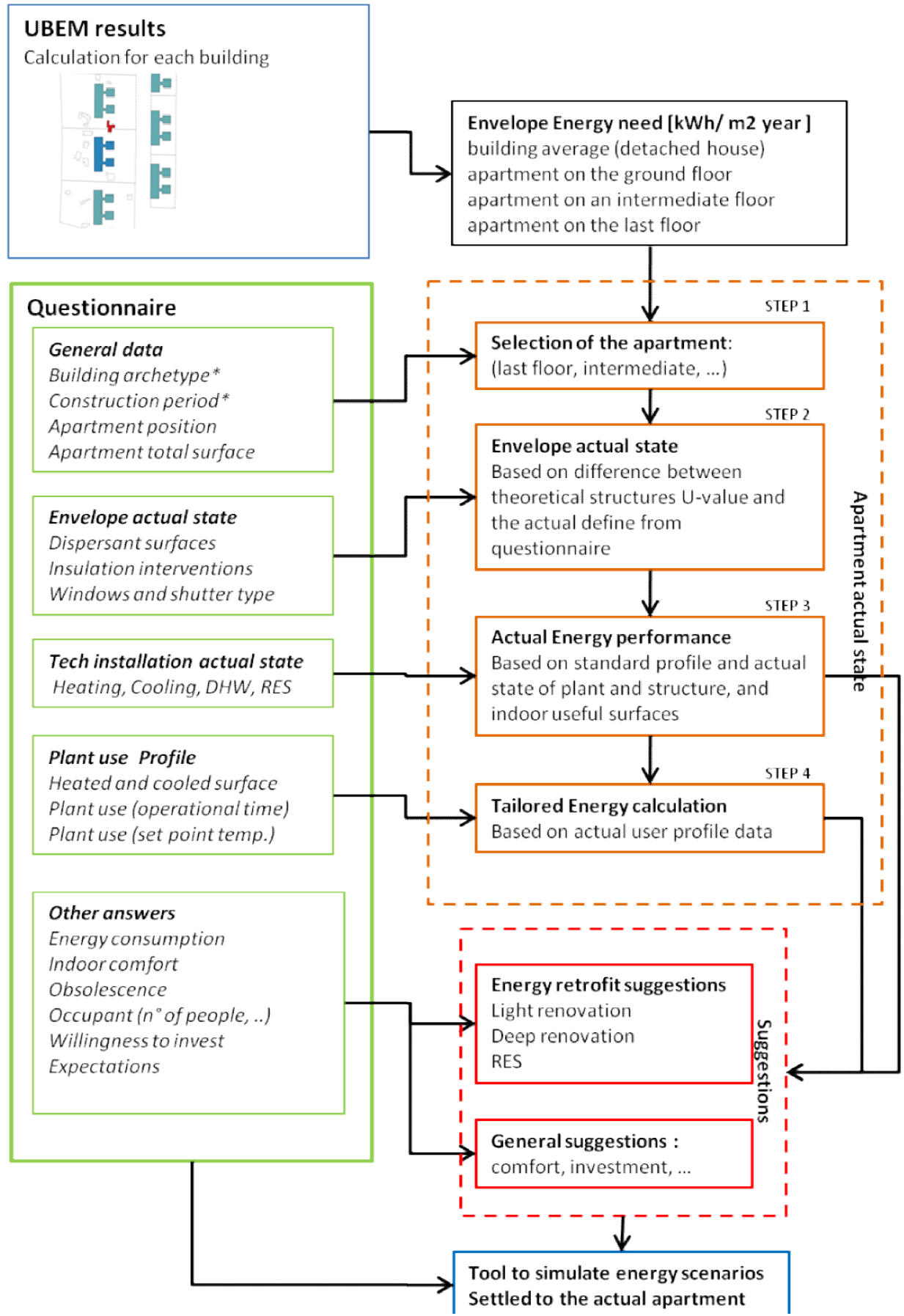

Fig. 3. Framework of the home occupant interface 


\section{Conclusions}

The AUREE project is still in progress and a partial version of portal that contains a beta of the house occupant interface with the questionnaire is already available. During the next year, a group of representative stakeholder will be involved in order to test all the interfaces and the others informative materials of the portal. In order to improve and the effectiveness of the home occupants interface, a key point will be the analysis of the flow of the questionnaire based data (quality, number, spatial distribution ...). The proposed methodology adopts some more related to local context base dataabout structures and typologies that could ensure a better significance of the results than other similar tools available in web. However, a typological approach with a level of simplification and hypothesisgreater than an ordinary design activity is still needed. Therefore, a research issue of the further experimentation is to define a balance between complexity of the procedure and of the interfaces and quality of the results that are still based on a priori assumptions related to UBEM procedure and on the reliability of the participatory-based data.

The assessment of the questionnaire based data will be useful to define and test the algorithms for step 4 (fig 3 ) and for the development of the tool for the scenarios definition. Also an improvement of steps 1-2-3 (fig 3) procedures will be evaluated and tested implementing more detailed and reliable calculation procedure.

The experimentation will be implemented on a part of the city of Carbonia a Sardinian (IT) average sized (about 28200 inh. ISTAT 2018)Mediterranean town. Carbonia is a fascist's new town (1938) that could be considered a proper context for the methodology experimentation because it is characterized by a rather than homogeneous built heritage about the material - constructive aspects.

The research framework and the article structure is to be attributed to Pili S., text has been wrote by Pili S,Poggi F. and Frau C., she is also the research groups supervisor. The resources for this publication has been given by the Sotacarbo SPA, within the "Research of Electric System" project funded by "Ministry of Economic Development" CUP: I34I19005780001

\section{References}

1. https://www.odyssee-mure.eu/publications/br/energy-efficiency-trends-policiesbuildings.pdf

2. ASHRAE Guideline 34P, Energy Guideline for Historical Buildings Second Public Review Draf, https://www.techstreet.com/ashrae/ashrae_standards.html

3. F. Ascione, N. Bianco, R.F. De Masi, F. De Rossi, G. P. Vanoli, "Energy retrofit of an educational building in the ancient centre of Benevento. Feasibility study of energy savings and respect of the historical value", Energy and Buildings Volume 95, 15 May 2015, Pages 172-183

4. L. Kranzl and the ENTRANZE consortium, Laying Down The Pathways To Nearly Zero-Energy Buildings A toolkit for policy makers, Final Report of the Policies to enforce the transition to nearly zero energy buildings in the EU-27 (ENTRANZE) founded by IEE (2014), http://www.entranze.eu/pub/pub-policies

5. https://app.enerfund.eu/

6. http://www.energiavalinta.fi/

7. https://oneplace.fbk.eu/ 
8. http://www.repowermap.org/

9. https://siape.enea.it/

10. https://homeenergysaver.lbl.gov/

11. https://www.greenovation.it/

12. http://sitmappe.comune.bologna.it/BolognaSolarCity/

13. https://www.enea.it/it/efficienza-energetica/condomini-4.0/

14. https://recon.smartenergycommunity.enea.it/

15. https://www.smarthome.enea.it/

16. C. Reinhart, C. Davila, Urban building energy modeling - A review of a nascent field, Building and Environment 97 (2016), 196-202. https://doi.org/10.1016/j.buildenv.2015.12.001

17. Lukas G. Swan, V. IsmetUrgursal, Modeling of end use Energy consumption in the residential sector: A review of modelling techniques, Renewable \& Sustainable Energy Reviews 13 (2009) 1819-1835.DOI:10.1016/j.rser.2008.09.033

18. Y. Q. Ang, M. Zachary, C. Berzolla, C.F.Reinhart From concept to application: A review of use cases in urban building energy modeling, Applied Energy Volume 279, 1 December 2020, https://doi.org/10.1016/j.apenergy.2020.115738

19. N. Abbasabadi, M. Ashayeri, Urban energy use modelling methods and tools: A review and an outlook, Building and Environment 161 (2019), 106 - 270, https://doi.org/10.1016/j.buildenv.2019.106270

20. R. Nouvel, A. Mastrucci, U. Leopold, O. Baume, V. Coors, U.Eicker, Combining GISbased statistical and engineering urban heat consumption models: Towards a new framework for multi-scale policy support, Energy and Buildings 107(2015), 204-212. https://doi.org/10.1016/j.enbuild.2015.08.021

21. J. Keirstead, M. Jennings, A. Sivakumar, A review of urban energy system models: Approaches, challenges and opportunities, Renewable and Sustainable Energy Reviews 16 (2012) 3847-3866; https://doi:10.1016/j.rser.2012.02.047

22. 1https://episcope.eu/building-typology/tabula-webtool/

23. Y. Chen, T. Hong, M.AcPiette, City-scale building retrofit analysis: A case study using CityBES, Build. Simul. (2017), San Francisco, CA, USA. https://citybes.lbl.gov/BS2017_CityBES_Paper_Final.pdf

24. C. Reinhart, T. Dogan, J. A. Jakubiec, T. Rakha, A. Sang, Umi - an urban simulation environment for building energy use, daylighting and walkability. In: Proc BS201313th conference int build performance simulation assoc; 2013. p. 476-83

25. J. Sokol, C. D. Cerezo, C. Reinhart, Validation of a Bayesian-based method for defining residential archetypes in urban building energy models. Energy and Buildings, Volume 134, 1 January 2017, Pages 11-24, https://doi.org/10.1016/j.enbuild.2016.10.050.

26. D.Lgs. 192/2005 (and s.m.s) and Italian Standard UNI/TS 11300:2014. https://www.cti2000.eu/la-uni-ts-11300/ 